\title{
Annonces de publications "d'Apadiens"
}

\section{(2) OpenEdition}

Journals

Édition électronique

URL : http://journals.openedition.org/apad/403

DOI : 10.4000/apad.403

ISSN : 1950-6929

Éditeur

LIT Verlag

Édition imprimée

Date de publication : 15 mars 1992

\section{Référence électronique}

"Annonces de publications "d'Apadiens" », Bulletin de l'APAD [En ligne], 3 | 1992, mis en ligne le 06 juillet 2006, consulté le 15 septembre 2020. URL : http://journals.openedition.org/apad/403

Ce document a été généré automatiquement le 15 septembre 2020.

Bulletin de I'APAD 


\section{Annonces de publications "d'Apadiens"}

Irrigators and engineers

Edited by Geert Diemer et Jacques Slabbers. Until the 1980s, irrigation development in Asia, Africa and South America was viewed by many as a hard-nosed, purely physical enterprise. In response to disappointing hardware performance, irrigation engineers developed a new consensus : not only plants, soil and water required attention, the management of canal networks needed it 100. It remains uncertain whether this insight is sufficient to make irrigation infrastructures more productive. It may be supposed that reasons for the poor performance of irrigation schemes must also be looked for in the design and implementation processes from which they originate. The essays in this book focus on both. A number of engineers and social scientists deconstruct current design method and related procedures for planning and implementation. Some add proposals for alternative procedures or report experiments, others focus on management issues. The essays were written in honour of Lucas Horst, Professor Emeritus of Irrigation at Wageningen Agricultural University.

\section{L'appropriation de la terre en Afrique noire}

4 E. Le Bris, E. Le Roy et P. Mathieu, (éditeurs), Manuel d'analyse, de décision et de gestion foncières, Paris, Karthala, 1991, 359 p. Prix Afrique : $60 \mathrm{FF}$, prix public : 150FF. Premier manuel sur la question foncière en Afrique, l'ouvrage répond à des questions que posent chercheurs et praticiens: quel facteur prime dans le pastoralisme ou l'arboriculture, qu'est-ce qu'un parcellaire, une trame foncière et comment user (sans abuser) du cadastre ? Il n'a pas réponse à tout, loin de là, car les retards de la recherche sont, parfois, "abyssaux". Mais cet ouvrage de recherche appliquée, mobilisant une trentaine de chercheurs, expose de manière transdisciplinaire les courants et les tendances de l'analyse contemporaine. $\mathrm{n}$ renoue ainsi avec certains débats (ceux d'AMIRA dans les années soixante-dix sur la "transition") et approfondit certaines réflexions (à propos de la gestion des ressources "communes", des conditions de la décentralisation foncière, ou des effets déstabilisants et insécurisants des programmes d'ajustements structurels...). Il explore également de nouvelles voies pour ce qui concerne le maintien de la fertilité des sols ou l'environnement. Avec pragmatisme, il 
veut montrer qu'il n'y a pas de bonne gestion foncière sans option politique clairement et démocratiquement affichée et que ces choix doivent reposer sur une connaissance et une prise en compte des besoins effectifs des populations en renonçant ainsi à des conceptions "prométhéennes" du développement.

5 CIDEP (centre international de formation et de recherche en population et développement en association avec les nations unies) publie des Cahiers et des Notes et Documents dont les auteurs sont souvent des "apadiens". Informations : C.I.D.E.P 1, place Montesquieu BP 17 B 1348 Louvain la Neuve.

Cahiers :

Cahier $\mathrm{n}^{\circ}$ 1: Nzisabira J., Accumulation du peuplement rural et ajustements agro-pastoraux au Rwanda, juin 1989, 76 p., 250 FB.

Cahier $\mathrm{n}^{\circ} 2$ : Singleton M., Mazombwe, l'Homme-lion. De la métamorphose magique à la manipulation génétique, juillet 1989, 86 p., 250 FB.

Cahier $n^{\circ} 3$ : Singleton M., Des casseurs de cailloux à Dakar : une classe ou une caste ? Jalons pour une enquête, octobre 1989,49 p., 200FB.

11 Cahier $\mathrm{n}^{\circ} 4$ : Loriaux M., La fin des certitudes: modèles démo-socio-économiques à réformer, systèmes informationnels à inventer, juillet 1990,48 p., 220 FB.

Cahier $n^{\circ} 5$ : Singleton M., (R)apports pédagogiques, août 1990, 125 p., 220 FB. l'ajustement, février 1991, 59 p., $275 \mathrm{FB}$ 
Farmer-managed irrigation in the Senegal valley : implications for the current design method

End-of project report, WARDA/Wageningen Agricultural University, Water Management Project. St-Louis and Wageningen. Diemer G. and F.P. Huibers (1991). Summary: this report is based on field research in the Senegal River valley. It shows how farmers manage irrigation schemes, relating management to scheme design. One chapter treats the characteristics and utilization of the relatively successful village irrigation schemes. Others are devoted to the concept of so-called intermediate schemes, which are larger in scale and designed with the market production of rice as the objective. Irrigation will only contribute to development if planners and engineers develop a better design method. This end-of-project report shows that designing effective irrigation schemes requires the integration of technical and socio-cultura1elements.

\section{Organisations paysannes au Sénégal}

Parmi les récentes publications de l'institut Allemand de Développement, nous avons noté : "Organisations paysannes au Sénégal: comment renforcer les structures intermédiaires dans le processus de transformation socio-économique?" par G. Lachenmann et al., et "Social movements and civil society in West Africa" par G Lachenmann. Ces ouvrages feront l'objet d'un compte-rendu dans le prochain bulletin.

Contact: Institut Allemand de développement, Fraunhoferstrasse 33-36 : D 1000 Berlin 10.

\section{Chroniques (réseaux technologie, culture et développement)}

Dans le numéro 54 (février 1992) nous avons noté un reportage sur le crédit rural au Burkina Faso (L. Falque- Vert), une enquête de Philippe Lavigne-Delville et Pierre Deschamps sur une coopérative fondée par des émigrés maliens de retour, un article d'E. Ndione sur une expérience de crédit auprès de vendeurs de poisson au Sénégal, un autre de Fatime NDiaye sur le foisonnement des structures associatives au Sénégal.

Contact: Chroniques, magazine trimestriel d'information, GRET, 213 rue La Fayette, 75010 Paris, France.

\section{Publications de la sorbonne}

1, rue Victor-Cousin - 75231 Paris Cedex 05. Vient de paraître : État et société dans le Tiers-monde. De la modernisation à la démocratisation? études réunies et présentées par Maxime Haubert, Christiane Frelin, François Leimdorfer, Alain Marie et Nam Trân Nguyên Trong. 